\title{
The effect of Rotation Speed on Cavitation of Small Flow Micro
}

\section{- High Speed Centrifugal Pump}

\author{
Shuai $\mathrm{Hu}^{\mathrm{a}}$, Wenwu Song ${ }^{\mathrm{b}}$, Qianyu $\mathrm{Xu}^{\mathrm{c}}$, Jianwei Shi ${ }^{\mathrm{d}}$ \\ (School of Energy and Power Engineering, Xihua University, Chengdu 610039, \\ China) \\ aking-insisths@foxmail.com, bwenwus@163.com, ' $1501569407 @ q q . c o m,{ }^{d} 57369036$ \\ 6@qq.com
}

Abstract: In order to improve the micro-high-speed centrifugal pump cavitation performance, to understand its internal cavitation characteristics. The internal flow field of the centrifugal pump model established by UG was simulated by CFX software. The performance of the centrifugal pump in different speed, flow and different inlet pressure conditions for performance analysis.The numerical results show that: The smaller the centrifugal pump speed, the smaller the head and efficiency. Impeller flow and the blade surface pressure along the inlet to the direction of the export gradually increased. The overall pressure loads distribution of the impeller increases with the increase of the rotational speed. The vacuoles first appeared at the impeller inlet and the blade suction surface. The smaller the speed, the smaller the amount of vacuoles produced. Overall, the more the speed away from the design speed, the worse the performance of centrifugal pumps and the impeller blade profile must be redesigned.

Key words: Micro high speed pump; Rotating speed; Small flow; Pressure; Number of bubbles.

\section{Introduction}

With the continuous progress of science and technology, centrifugal pumps at the forefront of precision machinery and other high-tech development. While requiring high-speed, high efficiency and high reliability and other properties. The micro-high-speed pump can be used in the field of robotics, space and medical equipment and other advanced scientific fields. So the micro-high-speed pump research should take into account the impact of internal cavitation. For the design of a higher performance centrifugal pump to provide data for reference.

Domestic and foreign scholars in the centrifugal pump cavitation research have made many contributions. The applicability of the Kunz model, the Zwart-Gerber-Belamri model and the Schnerr-Sauer model in the simulation of cavitation flow in centrifugal pumps are compared. Liu Houlin et al(2012). The unsteady flow and the influence of the volute tongue are analyzed of the centrifugal pump under non - design conditions. (ZHU Baoshan et al,2013; Meng Genqiqige et al, 2015). The influence of the impeller blades on the cavitation of the centrifugal pump is analyzed, and the results show that the medium and large centrifugal pump has the best performance for six blades. Yuan Shouqi et al, (2015). Both the results under the steady and transient cavitation have been discussed in a nuclear centrifugal pump. Fu Qiang et al, (2016). Application of the two-phase three-component computational model to predict cavitating flow in a centrifugal pump and its validation. Wang Yong et al, (2016).

In summary, this article mainly in the speed, import pressure and small flow and other design parameters, from the micro-high-speed pump external characteristics, cavitation characteristics, leaf and runner pressure load distribution and vacuolar birth and development of four aspects,The 
internal flow field and cavitation characteristics of a detailed analysis to provide accurate numerical basis for the theory.

\section{Computational model and meshing}

A low specific speed micro - high - speed centrifugal pump is used as the numerical analysis model. The main parameters of its design are: the design flow $Q_{\mathrm{d}}$ is $15 \mathrm{~m}^{3} / \mathrm{h}$; the design head $H$ is 42 meter; the rotation speed $n$ is $12000 \mathrm{r} / \mathrm{min}$; the impeller diameter $D_{1}$ is $0.055 \mathrm{~m}$; the impeller outlet width $b_{2}$ is $0.006 \mathrm{~m}$; the blade number $Z$ is 4 . Three-dimensional model is established and optimized by software CFturbo and UG. The whole model includes impeller inlet extension, impeller, volute and volute export extension of four parts, as shown in Figure 1.

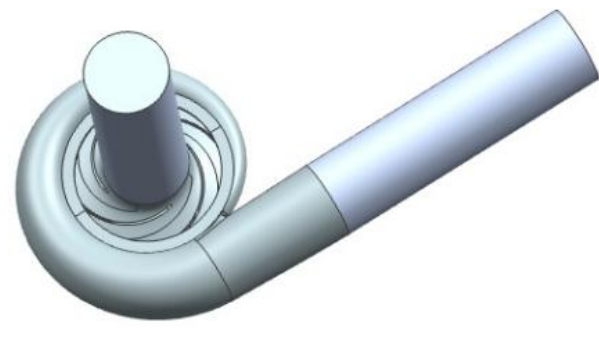

Fig1 Calculation model

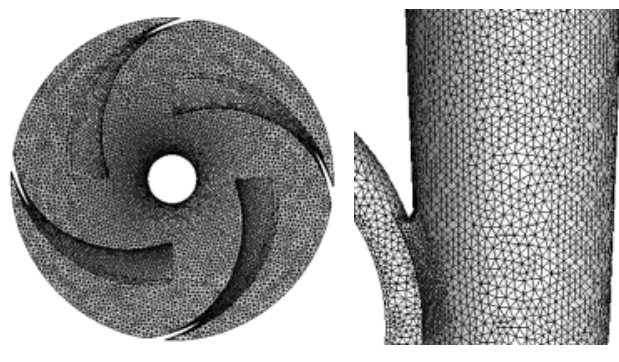

Fig2 Impeller and tongue part of the grid

The model was partitioned by ANSYS ICEM CFD software. As centrifugal pump blade and volute and other areas has a greater distortion. So the use of adaptability of non-structural tetrahedral meshes for flow channel division. In order to improve the accuracy of numerical simulation, the volute and the impeller interface, as well as the tongue position is grid encryption, as showed in Figure 2.

\section{Control equations and cavitation models}

The RNG k- $\varepsilon$ Turbulence model is used to predict the rotation of turbulent vortices and to predict the flow of high flow line and high strain rate more accurately. The cavitation model is based on the Rayleigh-Plesset equation homogeneous flow model provided by ANSYS CFX software, that is, the cavitation flow is usually regarded as a single-phase flow with average fluid properties to calculate the mass transfer process between the gas phase and liquid phase. The equation is:

$$
R_{B} \frac{\mathrm{d} 2 R_{B}}{\mathrm{~d} t^{2}}+\frac{3}{2}\left(\frac{\mathrm{d} R_{B}}{d t}\right)^{2}+\frac{2 S}{\rho_{1} R_{B}}=\frac{P_{s a t}-P}{\rho_{1}}
$$

Where: $R_{\mathrm{B}}$ is the bubble radius; $P_{\text {sat }}$ is saturated vapor pressure; $\rho_{1}$ is liquid density.

\section{Boundary condition and interface setting}

The CFX software was used to simulate the cavitation of small flow conditions and different rotating speed conditions. The import flow is set to total pressure and the outlet is mass flow; the grid node is adapted to the GGI mode; to 25 degrees Celsius of water and water vapor as the working medium. The calculation results of single - phase flow in the cavitation model are taken as the initial values of cavitation two - phase flow. The volume fraction of liquid at the inlet is 1 and the volume fraction of the gas phase is zero when the cavitation is simulated. Impeller leaves and upper and lower wheel wall surface with no slip wall, the rest of the wall are static without 
sliding wall. The exit of the impeller inlet and extension of the micro high speed pump is set to the Frozen Rotor mode on the surface between the impeller and the volute. The calculated residuals converge below $10^{-5}$.

\section{Results and discussions}

\subsection{Parameter definition}

In order to better study and analyze the numerical results, the pump outlet peripheral speed $\mathrm{u}_{2}$ is defined as $\mathrm{u}_{2}=2 \pi n D_{1} / 60$; the head coefficient $\psi$ as $\psi=H /\left(\mathrm{u}_{2}^{2} / 2 \mathrm{~g}\right)$; the cavatation number $\sigma$ as $\sigma=\left(\mathrm{P}_{\text {in }}-\mathrm{P}_{v}\right) /\left(0.5 \rho_{1} \mathrm{u}_{2}^{2}\right)$, with $\mathrm{P}_{\text {in }}$ and $\mathrm{P}_{v}$ being the total pressure at the pump inlet and steam saturated gasification pressure, respectively. The corresponding size of the pump is equal, then the similarity law formula becomes: $H_{2}=H_{1} /\left(n_{1} / n_{2}\right)^{2}, Q_{2}=Q_{1} /\left(n_{1} / n_{2}\right)$.

\subsection{Micro high speed pump external characteristics}

At design point of view as a basis for reference, at other speeds, the similarity of the conversion, as showed in table1.

\begin{tabular}{lccc}
\multicolumn{4}{l}{ Table 1 The parameters of the similar operating point at each speed } \\
\hline 转速 $n\left(\mathrm{r} \cdot \mathrm{min}^{-1}\right)$ & 12000 & 6000 & 2980 \\
\hline 流量 $Q\left(\mathrm{~m}^{3} \cdot \mathrm{h}^{-1}\right)$ & 4.1592 & 2.0796 & 1.0329 \\
扬程 $H(\mathrm{~m})$ & 42 & 10.5 & 2.5901 \\
\hline
\end{tabular}

Numerical simulations are carried out without any loss. It is obvious that the head increases with decreasing flow, as showed in fig3(a). The variation of the head with the flow rate is similar to the curve at each speed. Small flow situation has a positive slope phenomenon, that is, unstable phenomenon. Simultaneously, efficiency grows with increasing traffic, as showed in fig3(b). Three kinds of speed conditions, the maximum efficiency of almost the same, slightly higher than the design speed. The efficiency curve at each speed is not a similar parabola. Overall, in similar operating conditions, the higher the speed the more prone to cavitation.

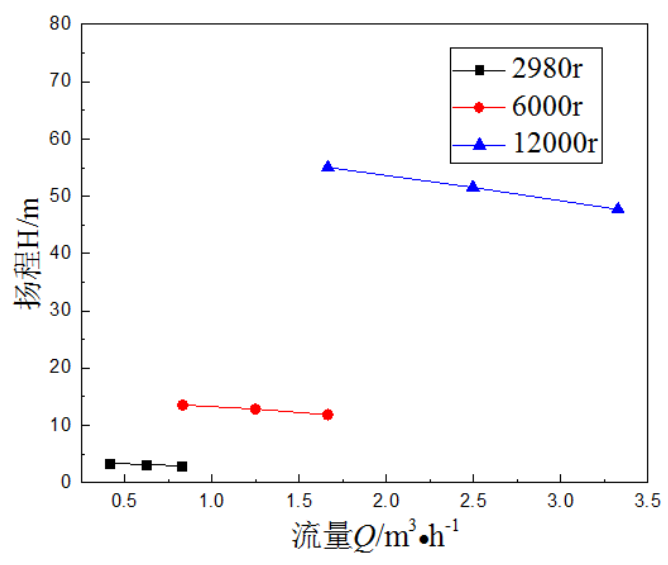

(a)

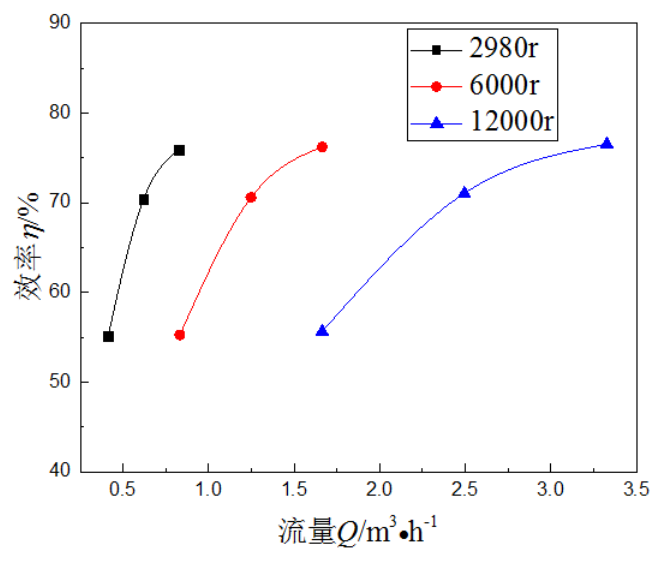

(b)

Fig3 The curve of external characteristic

\subsection{Cavitation external characteristic curve}

Fig.4, it is obvious that the head coefficient is increased with the decrease in flow under the same speed. Each curve tends to be smooth at the beginning. As the cavitation coefficient drops to a certain extent, all curves will appear to drop sharply. Simultaneously, the fluctuation curve appears before the steep drop. It is primarily because the number of vacuoles has increased dramatically and the volume fraction distribution in the impeller passage shows asymmetry. This 
situation due to the existence of the coupling effects between the impeller and volute. So making pressure distribution on the blade surface asymmetric that blocking the impeller flow path to destroy the pump performance. Moreover, the cavitation coefficient corresponding to the position of each curve occurring the steep drop is smaller. We can see that lower the rotation speed under different flow conditions, the corresponding steep drop position more concentrated and the better the cavitation performance.

The point at which the head coefficient is reduced by three percent is called the critical cavitation point, shown in fig5. Thus, the point corresponding of cavitation coefficient is the critical cavitation coefficient. It can be seen from the figure that the critical cavitation coefficient increases with increasing flow and the trend is roughly the same. When the speed is 12000 and 6000 rpm, their critical cavitation coefficient is not large, but with 2980 speed difference between the larger. Due to the speed is too small, resulting in the lack of power.

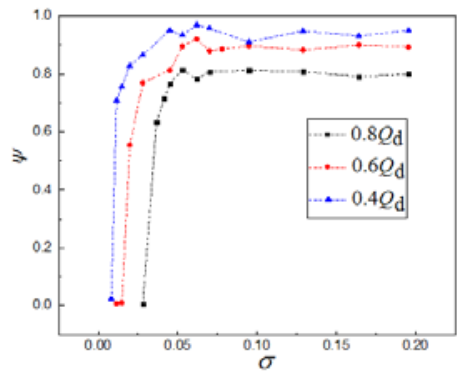

(a) $12000 \mathrm{r}$

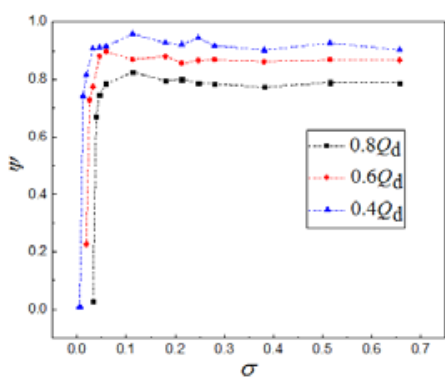

(b) $6000 \mathrm{r}$

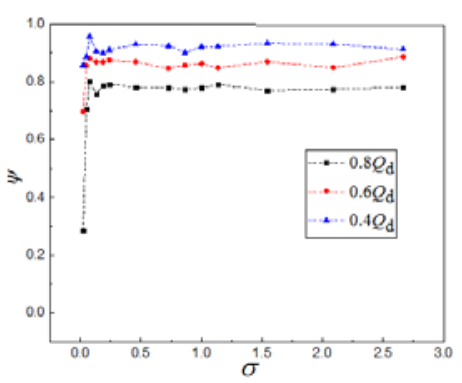

(c) $2980 \mathrm{r}$

Fig4 The variation coefficient of head coefficient with cavitation coefficient

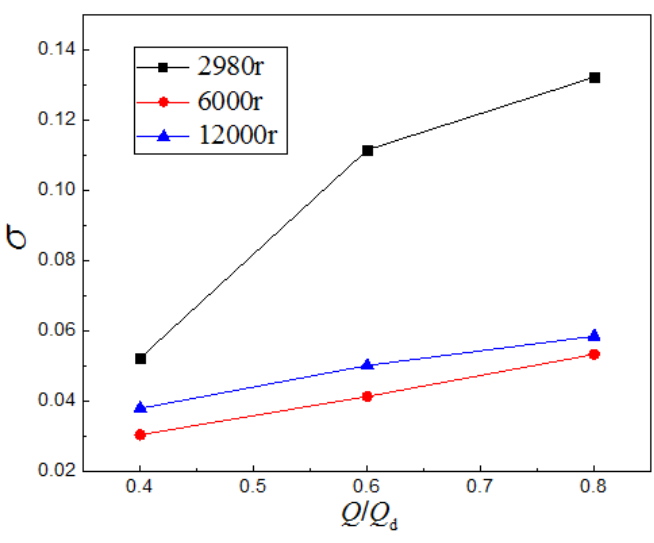

Fig5 Head down 3\% curve

\subsection{Blade surface pressure characteristics}

Under all operating conditions, the blade surface pressure load distribution always increases from the inlet and outlet, been displayed in Fig6. Meanwhile, the pressure on the suction side always increases and reached the maximum at the outlet. It is obvious that the occurrence of fluctuation on the leading edge of the blade suction side, shown in Fig6(a) -(b). It is mainly because the blade is nearest to the volute tongue, where the effect of the tongue is great and also the rotor-stator interaction.

In speed of 12000 and 0.6 times the design flow conditions, the pressure load is gradually decreased on the blade surface with the decreases of the cavitation coefficient, as showed in Fig6(a). It is not obvious that the variations of the pressure load on the blade pressure side under different flow, as showed in fig6(b). With the decrease of the rotational speed, the pressure load is 
gradually decreased on the blade surface, as showed in fig6(c).

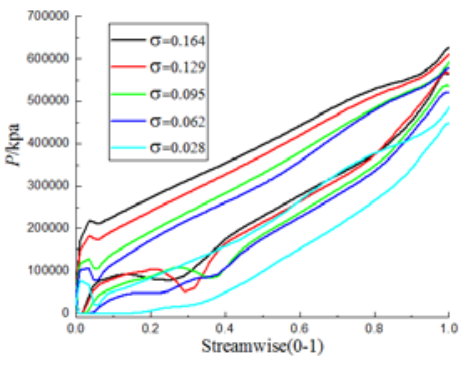

(a) $12000 \mathrm{r} 、 0.6 Q_{\mathrm{d}}$

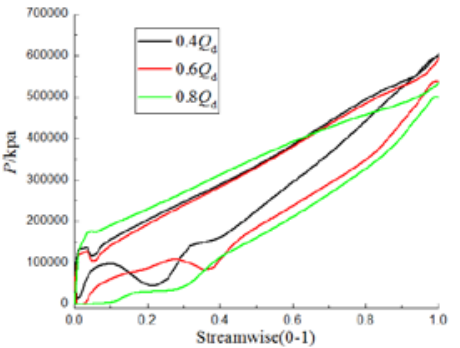

(b) $12000 \mathrm{r} 、 P_{\text {in }}=60000 \mathrm{~Pa}$

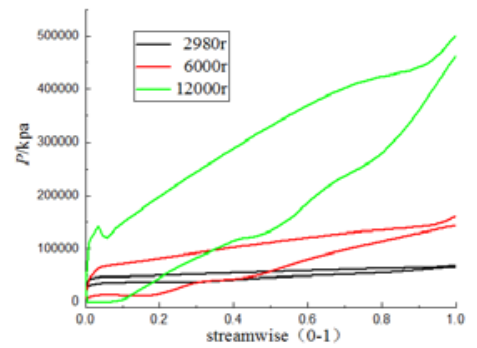

(c) $0.8 Q_{\mathrm{d}} 、 P_{\text {in }}=40000 \mathrm{~Pa}$

Fig6 pressure load distribution of the middle stearmline on the blade surface

\subsection{Impeller internal bubble characteristics}

Each speed in the flow of 0.8Qd similar operating point, the bubble volume fraction continues to increase as the inlet pressure decreases, shown in Fig7. At different speeds conditions, the vacuoles first appeared at the leading edge of the blade suction surface and expanded along the inlet to outlet. The vacuoles occupy almost the entire impeller flow passage when the inlet pressure is lower. Simultaneously, the maximum value of the bubble volume fraction exists in the middle of the blade suction surface near the hub.

The greater the rotational speed, the greater the inlet pressure when the impeller cavitation inception. In other words, the same import pressure conditions, the greater the speed, the more the amount of vacuoles generated. Moreover, the volume fraction distribution in the impeller passage shows asymmetry due to the pressure is irregular and the effect of the volute. Impeller internal pressure distribution asymmetry is more obvious, with the increase in speed.

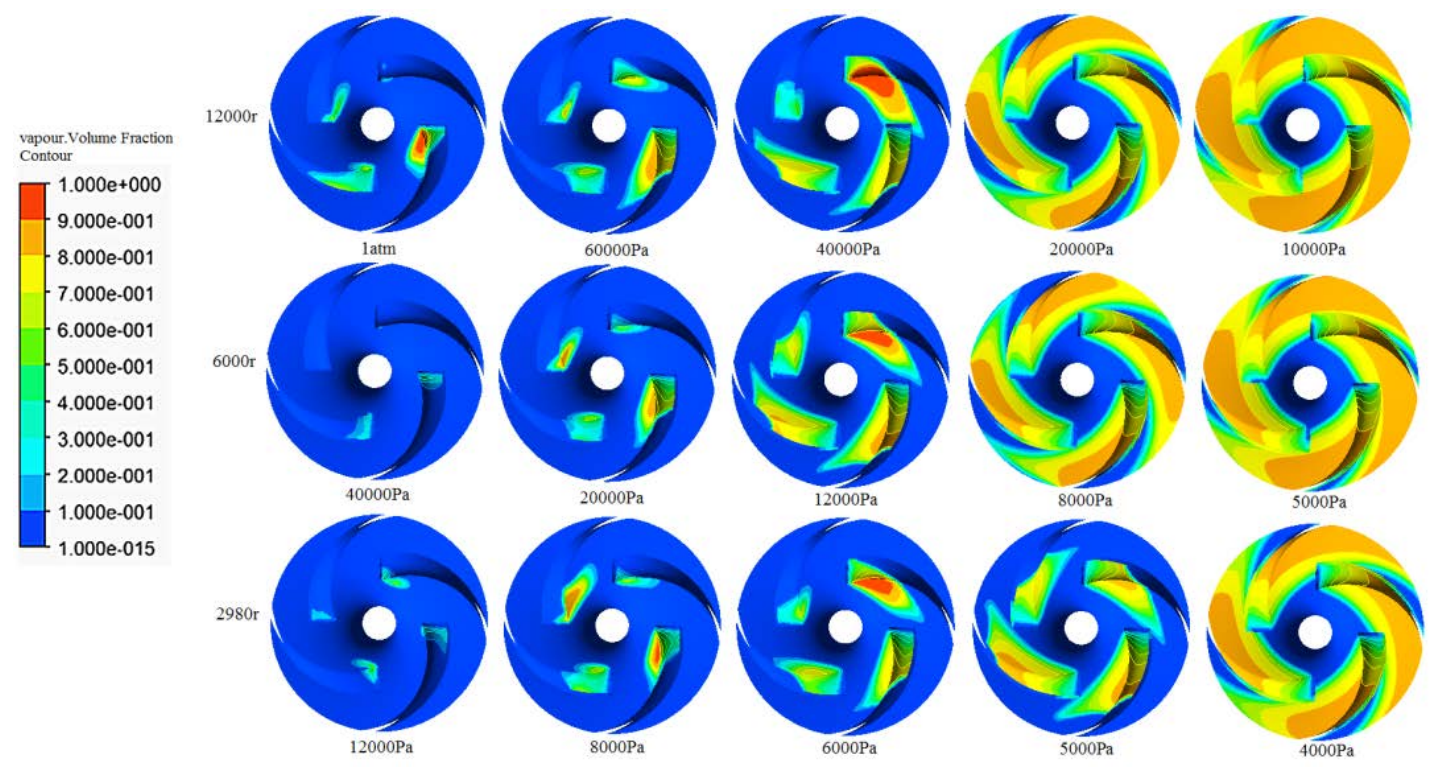

Fig7 Impeller internal vapor volume fraction change clouds under different import pressure

\section{Conclusions}

Deceleration has a negative impact on the volume and mechanical efficiency of micro high speed centrifugal pumps. The farther away from the rated speed, the more obvious the decline in efficiency. At the same time, the more the speed decreases, the more the head decreases accordingly. The smaller the speed, the greater the impact on the head coefficient of the 
centrifugal pump. The fluctuation of the head coefficient before the steep drop is caused by the uneven pressure distribution inside the impeller.

Rotating impeller and fixed volute tongue interaction between the static and dynamic effects, resulting in impeller blade and flow passage pressure asymmetry. The low pressure of the impeller flow passage and the blade is produced at the inlet and the suction side of the blade leading edge, respectively. Each speed of similar operating conditions, the pressure on the impeller flow path and the blade surface extends along the direction of the inlet to the outlet to the inlet pressure decreases. The smaller the speed, the smaller the overall pressure inside the impeller.

Under each speed condition, as the inlet pressure decreases, the volume of the cavities increases gradually along the impeller inlet to the outlet. Impeller internal cavitation phenomenon more and more serious with the increase in speed. In summary, it should be fully considered that the internal flow characteristics of the micro high speed centrifugal pumpcentrifugal pump, when changing the centrifugal pump speed larger case. At the same time, it is necessary to redesign the vane profile or change the blade structure. To improve the cavitation inside the centrifugal pumps performance and efficiency.

\section{Acknowledgements}

This work was financially supported by Science and Technology Department of the project of Sichuan Province(2016JY0187); Department of education major training project of Sichuan Province(14CZ0013); fluid and power machinery key laboratory of the Ministry of Education Fund (szjj2016-003). Corresponding author: Professor SONG Wenwu

\section{References:}

[1] Liu Houlin,Liu Dongxi,Wang Yong. et al. Applicative evaluation of three cavitating models on cavitating flow calculation in centrifugal pump[J]. Transactions of the Chinese Society of Agricultural Engineering, 2012,16: 54-59.

[2] Tan Lei, Zhu Baoshan, Cao Shuliang, et al. Numerical simulation of unsteady cavitation flow in a centrifugal pump at off-design conditions[J]. Proceedings of the Institution of Mechanical Engineers,Part C:Journal of Mechanical Engineering Science,2013,228(11):1994-2006.

[3] Meng Genqiqige,Tan Lei,Cao Shuliang,Wang Yu chuan. Numerical Simulation and Analysis of Unsteady Flow Characteristics in Centrifugal Pump Volute [J]. Chinese Journal of Mechanical Engineering, 2015, 22:183-190.

[4] Fu Yanxia,Yuan Shouqi,Yuan Jianping,et al. Effect of Blade Numbers on Cavitating Flow of Centrifugal Pump under Low Flow Rates[J]. Transactions of the Chinese Society for Agricultural Machinery,2015,04:21-27.

[5]Fu Qiang, Zhang F, Zhu RS, He B. A systematic investigation on flow characteristics of impeller passage in a nuclear centrifugal pump under cavitation state[J]. Annals of Nuclear Energy,2016,97:190-197.

[6]Wang Yong, Liu HL, Liu DX, et al. Application of the two-phase three-component computational model to predict cavitating flow in a centrifugal pump and its validation[J]. Computers and Fluids, 2016.131:142-150. 